\title{
CircZDHHC20 represses the proliferation, migration and invasion in trophoblast cells by miR-144/GRHL2 axis
}

Bing Zhou ${ }^{1 \dagger}$, Xia Zhang ${ }^{2 \dagger}$, Ting Li ${ }^{1}$, Rongping Xie ${ }^{3}$, Jianbin Zhou ${ }^{1}$, Yu Luo ${ }^{1}$ and Chunfen Yang ${ }^{4^{*}}$ (D)

\begin{abstract}
Background: Preeclampsia (PE) is a prevalent pregnancy disorder that has been one of the leading causes of maternal and perinatal mortality worldwide. Circular RNAs (circRNAs) have recently considered as important regulators in PE pathogenesis. In the current study, we aimed to explore the impact and mechanisms of circRNA zinc finger DHHCtype palmitoyltransferase 20 (circZDHHC20) in PE pathogenesis.

Methods: RNase R assay and reverse transcription with Oligo(dT) ${ }_{18}$ primers were performed to confirm that circZDHHC20 was indeed circular transcript. The expression of circZDHHC20, grainyhead-like 2 (GRHL2) and miR-144 were assessed by quantitative real-time polymerase chain reaction (qRT-PCR). Subcellular localization assay was used to determine whether circZDHHC20 was predominantly present in the cytoplasm. The target correlations between miR-144 and circZDHHC20 or GRHL2 were confirmed using dual-luciferase reporter and RNA immunoprecipitation (RIP) assays. Cell proliferation, migration, and invasion were detected by 3-(4,5-dimethylthiazol-2-yl)-5-(3carboxymethoxyphenyl)-2-(4-sulfophenyl)-2H-tetr-azolium (MTS), wound healing and transwell assays, respectively. Western blot was used for the quantification of GRHL2 protein level.

Results: Our data indicated that circZDHHC20 was up-regulated and miR-144 was down-regulated in PE placenta. CircZDHHC20 sequestered miR-144 by acting as a miR-144 sponge. CircZDHHC20 overexpression repressed trophoblast cell proliferation, migration, and invasion, while its knockdown exerted opposite effects. Moreover, miR-144 mediated the regulation of circZDHHC20 on trophoblast cell behaviors. GRHL2 was directly targeted and inhibited by miR-144. MiR-144 exerted regulatory effects on trophoblast cell proliferation, migration and invasion by GRHL2. Furthermore, circZDHHC20 modulated GRHL2 expression through sponging miR-144.
\end{abstract}

Conclusion: Our study suggested that a high level of circZDHHC2O inhibited the proliferation, migration, and invasion in trophoblast cells at least partially through sponging miR-144 and up-regulating GRHL2, providing a novel mechanism of PE pathogenesis.

Keywords: PE, circZDHHC20, miR-144, GRHL2, Trophoblast cells

*Correspondence: xatodm@163.com

${ }^{\dagger}$ Bing Zhou and Xia Zhang contributed equally to this work

${ }^{4}$ Department of Obstetrics and Gynecology, The First Affiliated Hospital,

University of South China, No. 69 Chuanshan Road, Hengyang 421001,

Hunan, China

Full list of author information is available at the end of the article

(c) The Author(s) 2020. This article is licensed under a Creative Commons Attribution 4.0 International License, which permits use, sharing, adaptation, distribution and reproduction in any medium or format, as long as you give appropriate credit to the original author(s) and the source, provide a link to the Creative Commons licence, and indicate if changes were made. The images or other third party material in this article are included in the article's Creative Commons licence, unless indicated otherwise in a credit line to the material. If material is not included in the article's Creative Commons licence and your intended use is not permitted by statutory regulation or exceeds the permitted use, you will need to obtain permission directly from the copyright holder. To view a copy of this licence, visit http://creativecommons.org/licenses/by/4.0/. The Creative Commons Public Domain Dedication waiver (http://creativecommons.org/publicdomain/zero/1.0/) applies to the data made available in this article, unless otherwise stated in a credit line to the data. 


\section{Highlights}

1. CircZDHHC20 sequestered miR-144 by acting as a miR-144 sponge.

2. A high level of circZDHHC20 inhibited the proliferation, migration, and invasion in trophoblast cells.

3. GRHL2 was a direct target of miR-144, and circZDHHC20 modulated GRHL2 exoression by sponging miR-144.

4. CircZDHHC20 regulated trophoblast cell behaviors through miR-144/GRHL2 axis.

\section{Background}

Preeclampsia (PE) is a prevalent pregnancy disorder that is often defined as new-onset hypertension and proteinuria during the second half of pregnancy $[1,2]$. PE affects approximately $5 \%$ of pregnant women and has been one of the leading causes of maternal and perinatal mortality worldwide. Normal proliferation, migration, and invasion of trophoblast cells are fundamental in maintaining the function of human placenta [3]. Inadequate trophoblast cell behavior may be associated with PE pathogenesis [4]. However, the underlying mechanisms of PE pathogenesis are still largely unknown.

Circular RNAs (circRNAs), a novel class of non-coding RNA transcripts, have a special circular covalently bonded structure without a $5^{\prime}$ cap or a $3^{\prime}$ polyadenylated (pA) tail [5]. Owing to the inherent stability by their circular structure and exonuclease resistance, circRNAs have been considered as excellent disease biomarkers [6]. Emerging evidence has shown that circRNAs serve as important regulators in PE pathogenesis. For instance, Deng et al. found that has_circ_0011460 might be a novel potential therapeutic target for severe PE treatment using RNA sequencing [7]. Zhou et al. [8] reported that circRNA pregnancy-associated plasma protein A (circPAPPA) was down-regulated in PE placenta, and its deficiency hampered trophoblast cell proliferation and invasion. Shen et al. [9] manifested that circRNA trinucleotide repeat containing 18 (circTNRC18) was up-regulated in PE placenta, and a high circTNRC18 expression repressed the migration and epithelial-mesenchymal transition (EMT) of trophoblast cells in PE. Moreover, recent research demonstrated that circRNA zinc finger DHHC-type palmitoyltransferase 20 (circZDHHC20, hsa_circ_0006732) was up-regulated in maternal blood cells of PE patients, eliciting its potential involvement in PE pathogenesis [10]. Nevertheless, the impact and molecular mechanisms of circZDHHC20 in PE pathogenesis remain indistinct.
MicroRNAs (miRNAs) are endogenous small noncoding transcripts that have been widely accepted to play critical roles in the development of PE [11, 12]. Previous researches had described that miR-144 was low expressed in PE placenta and maternal plasma [13, 14], and miR-144 overexpression enhanced trophoblast cell proliferation, migration, and invasion [15]. In recent years, the competing endogenous RNA (ceRNA) hypothesis suggests that circRNAs could functionally interact with miRNAs, illuminating the importance of such interaction in PE pathogenesis $[16,17]$. Intriguingly, two putative binding sites of miR-144 and circZDHHC20 (hsa_circ_0029698) or grainyhead-like 2 (GRHL2) were identified by the computational methods, eliciting the possibly regulatory network of the circZDHHC20/miR-144/GRHL2 axis. In the current study, we aimed to explore the impact of circZDHHC20 in PE pathogenesis and underlying mechanisms governing it.

\section{Materials and methods \\ Clinical specimens and cell culture}

Specimen collection and analysis processes were approved by the Ethics Committee of The Second Affiliated Hospital, University of South China. For this study group, placental tissues were collected from 15 healthy volunteers following a normal course of pregnancy and 26 PE patients, who underwent delivery at the Department of Obstetrics and Gynecology of The Second Affiliated Hospital, University of South China. Women with inflammatory disease, cardiovascular disease, chronic renal disease, diabetes mellitus, or recent infection were excluded from this study. All participators were asked to sign and confirm the written informed consent.

Human extravillous trophoblast HTR-8/SVneo cells were obtained from the American Type Culture Collection (ATCC, Manassas, VA, USA) and grown at $37{ }^{\circ} \mathrm{C}$ in an atmosphere of $5 \% \mathrm{CO}_{2} / 95 \%$ air in Dulbecco's Modified Eagle Medium/Nutrient Mixture F-12 (DMEM/F-12, Gibco, Tokyo, Japan) containing $10 \%(\mathrm{v} / \mathrm{v})$ fetal calf serum (FCS, Gibco), $100 \mathrm{U} / \mathrm{mL}$ penicillin, and $100 \mu \mathrm{g} / \mathrm{mL}$ streptomycin.

\section{RNA extraction and RNase $R$ digestion}

Total RNA was obtained using TRIzol reagent (Ambion, Thermo Fisher Scientific, Paisley, UK) from placental tissues and HTR-8/SVneo cells. In RNase R assay, RNA extracts $(100 \mu \mathrm{g})$ were incubated with RNase $\mathrm{R}(3 \mathrm{U} / \mu \mathrm{g}$, Epicenter Technologies, Madison, WI, USA) at $37^{\circ} \mathrm{C}$ for $20 \mathrm{~min}$, followed by the purification using the RNeasy MinElute Cleanup Kit (Qiagen, Tokyo, Japan), referring to the producer's guidance. 


\section{Reverse transcription and quantitative real-time polymerase chain reaction}

Random or Oligo $(\mathrm{dT})_{18}$ primers were used for reverse transcription polymerase chain reaction (PCR) with the RNA to cDNA EcoDry Premix Kit (Clontech, Otsu, Japan). The expression of circZDHHC20, linear ZDHHC20 mRNA, and GRHL2 mRNA were analyzed by quantitative real-time PCR (qRT-PCR) using SYBR Green PCR Kit (Qiagen) on a Rotor-Gene Q real-time PCR System (Qiagen), with the housekeeping gene $18 \mathrm{~S}$ rRNA as the internal control. The quantification of miR144 was carried out using the miScript II Reverse Transcription Kit (Qiagen) and miScript SYBR Green PCR Kit (Qiagen), and U6 level was used as the loading control. PCR primers used for PCR amplification were listed: circZDHHC20 sense: 5'-CCTACATTGACATGTACA CAGAACA-3', circZDHHC20 antisense: 5'-TTCCAC TGATCATTTTCTTGC-3'; linear ZDHHC20 mRNA sense: 5'-CGGCAACCCCTTTATGACTA-3', linear ZDHHC20 mRNA antisense: 5'-CCACTCACTGGA AGCAATCA-3'; miR-144 sense: 5'-ATCCAGTGCGTG TCGTG-3', miR-144 antisense: 5'-TGCTTATACAGT ATAGATG-3'; GRHL2 mRNA sense: 5'-GGGCATAGG ACTCCAGAGTAGGAA-3', GRHL2 mRNA antisense: 5'-TAGGGCAGGACTGGCAAACA-3'; $18 \mathrm{~S}$ rRNA sense: 5'-GGTCCGTGTTTCAAGACGG-3', 18S rRNA antisense: 5'-GCATATCAATAAGCGGAGGAA-3'; U6 sense: $5^{\prime}$-GCTTCGGCAGCACATATACTAA- $3^{\prime}$, U6 antisense: $5^{\prime}$-AACGCTTCACGAATTTGCGT-3'.

\section{Subcellular fractionation}

Cytoplasmic and Nuclear RNA Purification Kit (Norgen Biotek, Thorold, ON, Canada) was used to isolate and purify the RNA from nuclear and cytoplasm fractions. After that, the expression levels of circZDHHC20, $18 \mathrm{~S}$ rRNA and U6 in nuclear and cytoplasm fractions of HTR-8/SVneo cells were assessed by qRT-PCR assay.

\section{Cell transfection}

The sequences of circZDHHC20 and GRHL2 were cloned into the pcDNA3.1 vector (Promega, Toyko, Japan) to construct the corresponding overexpression plasmid, respectively, and nontarget pcDNA3.1 vector was used as the negative control. The modified miR-144 mimic (5'-UCAUGUAGUAGAUAUGACAU-3'), mimic negative control (miR-con, 5'-UUCUCCGAACGUGUC ACGUUU-3'), inhibitor of miR-144 (anti-miR-144, 5'-AUGUCAUAUCUACUACAUGA-3'), inhibitor negative control (anti-miR-con, $5^{\prime}$-CAGUACUUUUGUGUA GUACAA-3'), siRNA against circZDHHC20 (si-circZDHHC20, 5'-AAAAUACCAGUCUAUAAUGUU-3') and GRHL2 (si-GRHL2, 5'-AGGUAAUUCUGCUUUUCC GUC-3'), and a scrambled negative control sequence (si-con, 5'-UUCUCCGAACGUGUCACGU-3') were obtained from GenePharma (Shanghai, China). The Lipofectamine $^{\mathrm{TM}} 3000$ Transfection Reagent was purchased from Thermo Fisher Scientific and used for each transfection following the protocols of manufacturers.

\section{Bioinformatics}

Analysis for the directly interacted miRNAs of circZDHHC20 was carried out using the website tool Circular RNA Interactome (CircInteractome) (https://circintera ctome.nia.nih.gov/miRNA_Target_Sites/mirna_targe t_sites.html). The potential molecular targets of miR-144 were identified using the DIANA-microT-CDS server (http://diana.imis.athena-innovation.gr/DianaTools/ index.php? $r=$ microT_CDS/index).

\section{Dual-luciferase reporter assay}

The specific sequences of circZDHHC20 and GRHL2 $3^{\prime}$-untranslated region ( $3^{\prime}$-UTR) harboring the complementary site of miR-144 were cloned into the pmirGLO vector (Promega) to generate the corresponding wildtype luciferase reporter constructs (circZDHHC20-WT and GRHL2-WT), respectively. To generate circZDHHC20 and GRHL2 mutant-type reporter constructs, the Q5 Site-directed Mutagenesis Kit (New England Biolabs, Ipswich, MA, USA) was used to mutate the target site. After that, the constructs were introduced into HTR-8/SVneo cells, respectively, together with miR-con mimic, miR-144 mimic, anti-miR-con, or anti-miR-144. $24 \mathrm{~h}$ later, the cells were solubilized and luciferase assays were implemented using the PicaGene Dual SeaPansy Luminescence Kit (Toyo Inki, Tokyo, Japan) and an ARVO X4 plate reader (PerkinElmer, Yokohama, Japan).

\section{RNA immunoprecipitation (RIP) assay}

HTR-8/SVneo cells were homogenized in RIPA lysis buffer (Elabscience, Wuhan, China) supplemented with protease inhibitor cocktails (Thermo Fisher Scientific). Cell lysates were incubated with magnetic bead-coupled anti-Argonaute2 antibody (anti-Ago2, Abcam, Cambridge, UK) or a negative control IgG antibody (Abcam), following the instructions of the Magna RNA Immunoprecipitation Kit (Millipore, Zug, Switzerland). Total RNA was isolated from the beads and subjected to qRTPCR for the enrichment of circZDHHC20, miR-144, and GRHL2.

\section{Cell proliferation assay}

HTR-8/SVneo cells were seeded into 96-well plates and transfected with the indicated oligonucleotides or/and plasmids for $0,24,48$ and $72 \mathrm{~h}$. At each time point, the analysis of cell proliferation was performed by adding $20 \mu \mathrm{l}$ of 3-(4,5-dimethylthiazol-2-yl)-5-(3- 
carboxymethoxyphenyl)-2-(4-sulfophenyl)-2H-tetr-azolium (MTS) reagent provided by the CellTiter $96^{\circledR} \mathrm{AQ}$ ueous One Solution Cell Proliferation Assay Kit (Promega). The number of viable cells was proportional to the absorbance at $490 \mathrm{~nm}$ which was read by the FLUOstar Omega microplate reader (BMG Labtech, Ortenberg, Germany).

\section{Wound healing assay}

Migration ability of HTR-8/SVneo cells after transfection was assessed using a wound healing assay. In brief, cells $\left(1.0 \times 10^{6}\right)$ were placed in a 6 -well plate and subjected to various transfections. Then, a scratch was made in the cell monolayer using a sterile $200-\mu$ l pipette tip. The images of 0 and $48 \mathrm{~h}$ were photographed by an inverted microscope (Eclipse TE200, Nikon, Toyko, Japan) at $100 \times$ magnification. Lastly, the Image-ProPlus digitizing system (Media Cybernetics, Buckinghamshire, UK) was used to detect the distance of cell migration.

\section{Transwell invasion assay}

Inserts containing 8- $\mu \mathrm{m}$ pores in 24-transwell plates (BD Biosciences, North Ryde, NSW, Australia) were coated with Matrigel (BD Biosciences). Next, transfected cells $\left(5.0 \times 10^{4}\right)$ in serum-free media were seeded into the Matrigel-precoated inserts, and $500 \mu \mathrm{l}$ grown medium plus $10 \%$ FCS was added into the lower chamber. The cells that invaded through the pores of inserts were fixed with methanol and stained with $1 \%$ crystal violet. A $200 \times$ Nikon magnification microscope was used to photograph the invaded cells in three random fields.

\section{Western blot for GRHL2 level}

Transfected HTR-8/SVneo cells were lysed in RIPA lysis buffer as described above, and total protein was quantified by using a BCA Protein Assay Kit (Thermo Fisher Scientific). Protein extracts $(\sim 100 \mu \mathrm{g})$ were electrophoresed on an $8 \%$ SDS polyacrylamide gel, transferred to the nitrocellulose membranes (Amersham, Buckinghamshire, UK), and blocked in $3 \%(\mathrm{w} / \mathrm{v})$ non-fat dry milk in PBS. For primary antibodies, we used rabbit anti-GRHL2 antibody (1:1000, Abcam) or rabbit anti- $\beta$ actin antibody (1:5000, Abcam). Horseradish peroxidase (HRP)-conjugated goat anti-rabbit IgG (1:10,000, Abcam) was used as the secondary antibody. Immunoreactive bands were determined using the Enhanced Chemiluminescence Detection Kit (Millipore).

\section{Statistical analysis}

Significance between two data was analyzed by a twotail Student's $t$ test. Multiple group experiments were compared using one-way analysis of variance (ANOVA), followed by Bonferroni's multiple comparison test.
Correlations between circZDHHC20, miR-144 and GRHL2 expression in placental tissues from PE patients using the Spearman test. All results were reported as mean \pm standard deviation (SD). Statistical significance is denoted by ${ }^{*} P<0.05$.

\section{Results}

CircZDHHC20 was up-regulated and miR-144 was down-regulated in placental tissues from $P E$ patients Firstly, we determined the expression pattern of circZDHHC20 in placental tissues from PE patients and healthy volunteers. As shown by qRT-PCR, circZDHHC20 level was higher in PE group than that in control group (Fig. 1a). To confirm that circZDHHC20 was indeed circular transcript, RNase R assay was performed. These results revealed that linear transcript was significantly digested by RNase $\mathrm{R}$ and circZDHHC20 was resistant to RNase $\mathrm{R}$ digestion (Fig. 1b). Because circRNAs were depleted in the $3^{\prime} \mathrm{pA}$ tail, we used Random and Oligo $(\mathrm{dT})_{18}$ primers in reverse transcription experiments, respectively. As expected, circZDHHC20 level was lower compared with linear transcript (Fig. 1c). Additionally, the data of subcellular localization assay showed that circZDHHC20 was highly enriched in the cytoplasm fraction in HTR-8/SVneo cells (Fig. 1d). qRTPCR results also demonstrated that miR-144 expression was prominently reduced in placental tissues from PE patients compared to those of healthy volunteers (Fig. 1e). Besides, an inverse correlation between circZDHHC20 level and miR-144 expression was found in PE placental tissues (Fig. 1f).

\section{CircZDHHC20 sequestered miR-144 by acting as a miR-144 sponge}

CircRNAs prominently located in the cytoplasm are considered to regulate the abundance of available miRNAs through sponging miRNAs $[16,18]$. Herein, we further observed whether circZDHHC20 could act as miRNAs sponges. Using CircInteractome computational method, a putative complementary site for miR-144 was found in circZDHHC20 (Fig. 2a). To verify whether circZDHHC20 served as a molecular sponge of miR-144, dualluciferase reporter and RIP assays were implemented in HTR-8/SVneo cells. In dual-luciferase report assay, we cloned the partial sequences of circZDHHC20 harboring the miR-144-binding site into a luciferase plasmid and mutated the miR-144-binding site. In comparison to a corresponding negative control, the luciferase activity of wild-type reporter was significantly reduced by miR144 overexpression, while it was highly elevated after miR-144 deficiency (Fig. 2b, c). However, when the target site was mutated, little change in luciferase was observed with either miR-144 overexpression or knockdown 

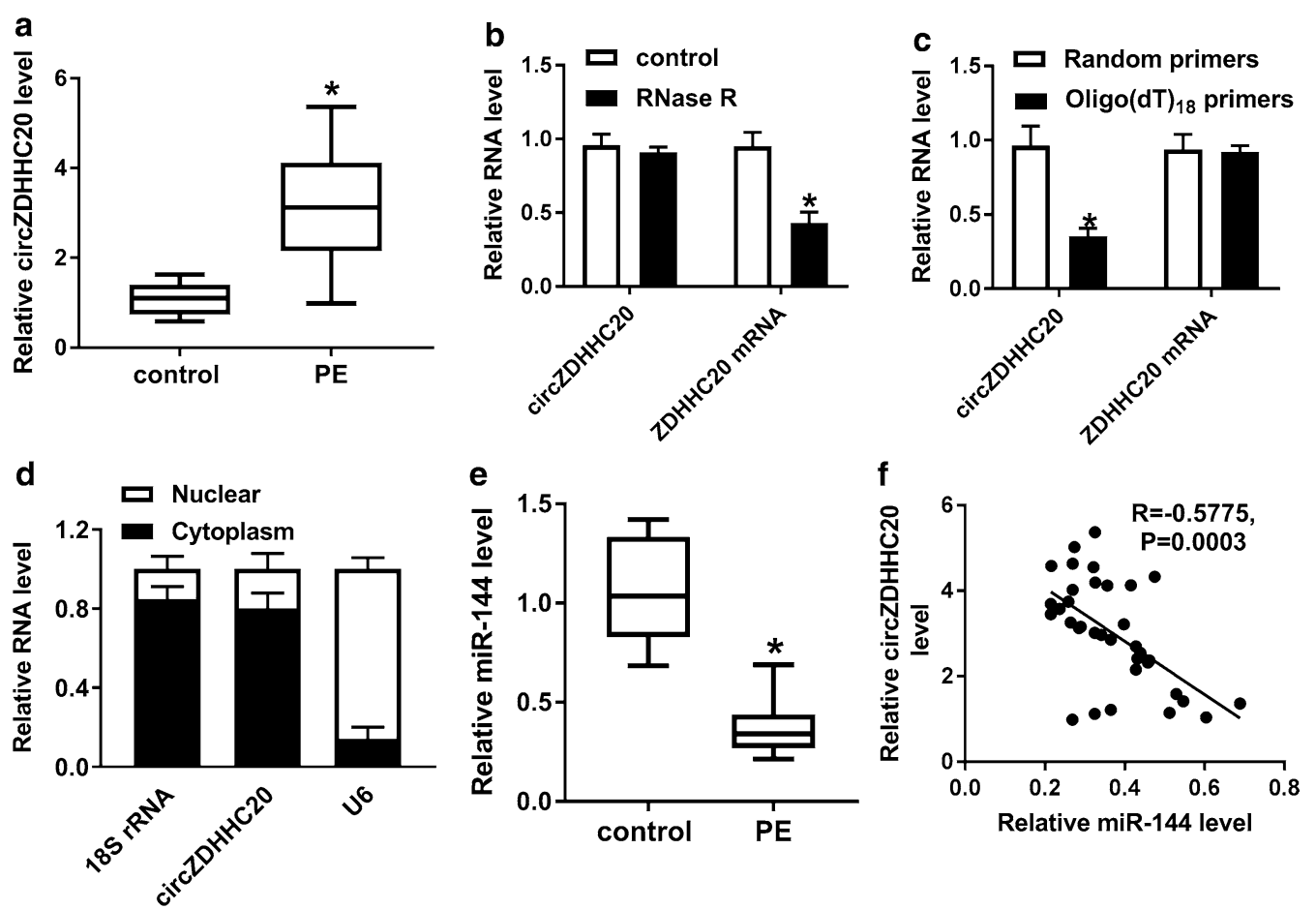

Fig. 1 CircZDHHC20 was up-regulated and miR-144 was down-regulated in PE placental tissues. a qRT-PCR for circZDHHC20 expression in placental tissues from 26 PE patients and 15 healthy volunteers. b qRT-PCR for the levels of circZDHHC20 and linear ZDHHC20 mRNA after RNase R digestion. c The expression of circZDHHC20 and linear ZDHHC20 mRNA by qRT-PCR in reverse transcription using Random and Oligo(dT) 18 Primers. d CircZDHHC20 level by qRT-PCR in the nuclear and cytoplasm fractions of HTR-8/SVneo cells. 18S rRNA and U6 were used as internal controls. e The level of miR-144 in placental tissues from 26 PE patients and 15 healthy volunteers. $\mathbf{f}$ The correlation between circZDHHC20 level and miR-144 expression in placental tissues from 26 PE patients using the Spearman test. ${ }^{*} P<0.05$

(Fig. 2b, c). In RIP experiments, anti-Ago2 antibody was used to bind to the RNA-induced silencing complex (RISC) [19]. These results revealed that circZDHHC20 and miR-144 were simultaneously enriched by anti-Ago2 antibody compared with IgG antibody (Fig. 2d), eliciting a possibility that circZDHHC20 could bind to miR-144 in the RISC. Moreover, the data of qRT-PCR showed that in contrast to their counterparts, miR-144 expression was prominently decreased by circZDHHC20 overexpression and increased when circZDHHC20 depletion in HTR-8/ SVneo cells (Fig. 2e), suggesting that the miR-144-binding site was functional.

\section{MiR-144 mediated the regulation of circZDHHC20 on proliferation, migration and invasion in trophoblast cells}

To explore the functional role of circZDHHC20 in PE pathogenesis, we manipulated its expression by siRNA targeting circZDHHC20 (si-circZDHHC20) and circZDHHC20 overexpression plasmid. MTS assays revealed that compared with a corresponding control, cell proliferation was significantly enhanced by circZDHHC20 knockdown (Fig. 3a) and weakened when circZDHHC20 up-regulation (Fig. 3b). Moreover, the results of wound healing and transwell assays showed that circZDHHC20 silencing led to the significant promotion in cell migration and invasion, while its overexpression exhibited opposite effects (Fig. 3c-f).

Then, we determined whether circZDHHC20 regulated trophoblast cell proliferation, migration, and invasion by miR-144. As expected, in contrast to their counterparts, the regulatory effects of circZDHHC20 knockdown and overexpression on cell proliferation, migration and invasion were significantly abolished by the introduction of anti-miR-144 or miR-144 mimic in HTR-8/SVneo cells (Fig. 3a-f).

\section{GRHL2 was directly targeted and inhibited by miR-144}

Next, using microT-CDS online software, a predicted miR-144-binding site was identified in the 3'-UTR of GRHL2 (Fig. 4a). To validate whether GRHL2 was a direct target of miR-144, we carried out dual-luciferase reporter and RIP assays. As shown in Fig. 4b, c, the luciferase activity of GRHL2 3'-UTR reporter construct was significantly down-regulated by miR-144 

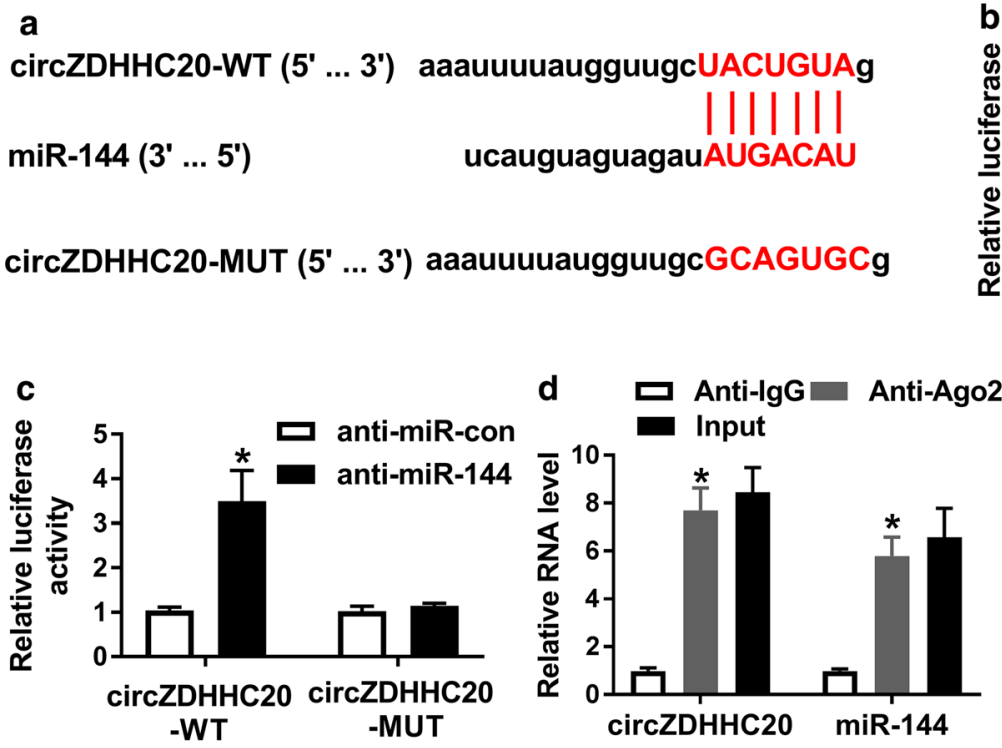

Fig. 2 CircZDHHC20 acted as a molecular sponge of miR-144. a Schematic of circZDHHC20 illustrating position of the miR-144-binding site and mutated the miR-144-binding site. b, c Relative luciferase activity in HTR-8/SVneo cells cotransfected with circZDHHC20-WT or circZDHHC20-MUT and miR-con mimic, miR-144 mimic, anti-miR-con or anti-miR-144. d The enrichment of circZDHHC20 and miR-144 in the RISC of HTR-8/SVneo cells using anti-Ago2 or lgG antibody, with Input content as positive control. e The expression of miR-144 by qRT-PCR in HTR-8/SVneo cells transfected with negative control plasmid (vector), circZDHHC20 overexpression plasmid (circZDHHC20), si-con or si-circZDHHC20. * $P<0.05$

overexpression and up-regulated following miR-144 silencing. However, these effects were highly abrogated by the mutation of the seed region (Fig. 4b, c). Moreover, anti-Ago2 antibody synchronously induced the significant enhancement in the enrichment of GRHL2 and miR-144 (Fig. 4d). The data of qRT-PCR assay also demonstrated a strong up-regulation of GRHL2 mRNA level in PE placental tissues compared with the healthy group (Fig. 4e). Moreover, GRHL2 protein level was strikingly reduced by a high miR-144 expression, while it was prominently elevated after miR-144 silencing in HTR-8/SVneo cells (Fig. 4f).

\section{The regulatory effects of miR-144 on trophoblast cell proliferation, migration and invasion were reversed by GRHL2 expression alteration}

To provide further mechanistic insight into the link between miR-144 and GRHL2 in PE pathogenesis, HTR-8/SVneo cells were transfected with miR144 mimic + GRHL2 overexpression plasmid or anti-miR-144+si-GRHL2. In comparison to the negative group, cell proliferation was highly facilitated following miR-144 overexpression (Fig. 5a) and hindered by a low expression of miR-144 (Fig. 5b). Furthermore, miR-144 overexpression resulted in the enhancement in cell migration and invasion, while miR-144 depletion showed opposite effects (Fig. 5c-f). More interestingly, the regulatory effects of miR-144 overexpression and depletion were strongly abolished by the contransfection of GRHL2 overexpression plasmid or si-GRHL2 (Fig. 5a-f).

\section{CircZDHHC20 protected against GRHL2 repression through sequestering miR-144}

Lastly, we investigated whether, if so, how circZDHHC20 modulated GRHL2 expression in HTR-8/SVneo cells. qRT-PCR results revealed that GRHL2 expression was inversely correlated with miR-144 level and positively correlated with circZDHHC20 expression in placental tissues from PE patients (Fig. 6a, b). As expected, in comparison to their counterparts, GRHL2 protein expression was significantly increased by highly expressed circZDHHC20 (Fig. 6c), while it was remarkably decreased when circZDHHC20 deficiency (Fig. 6d). Nevertheless, these effects were strongly reversed in response to miR144 level alteration (Fig. 6c, d).

\section{Discussion}

It has become increasingly clear that many circRNAs are abnormally expressed in PE placenta, providing a novel avenue of research regarding the pathogenesis 


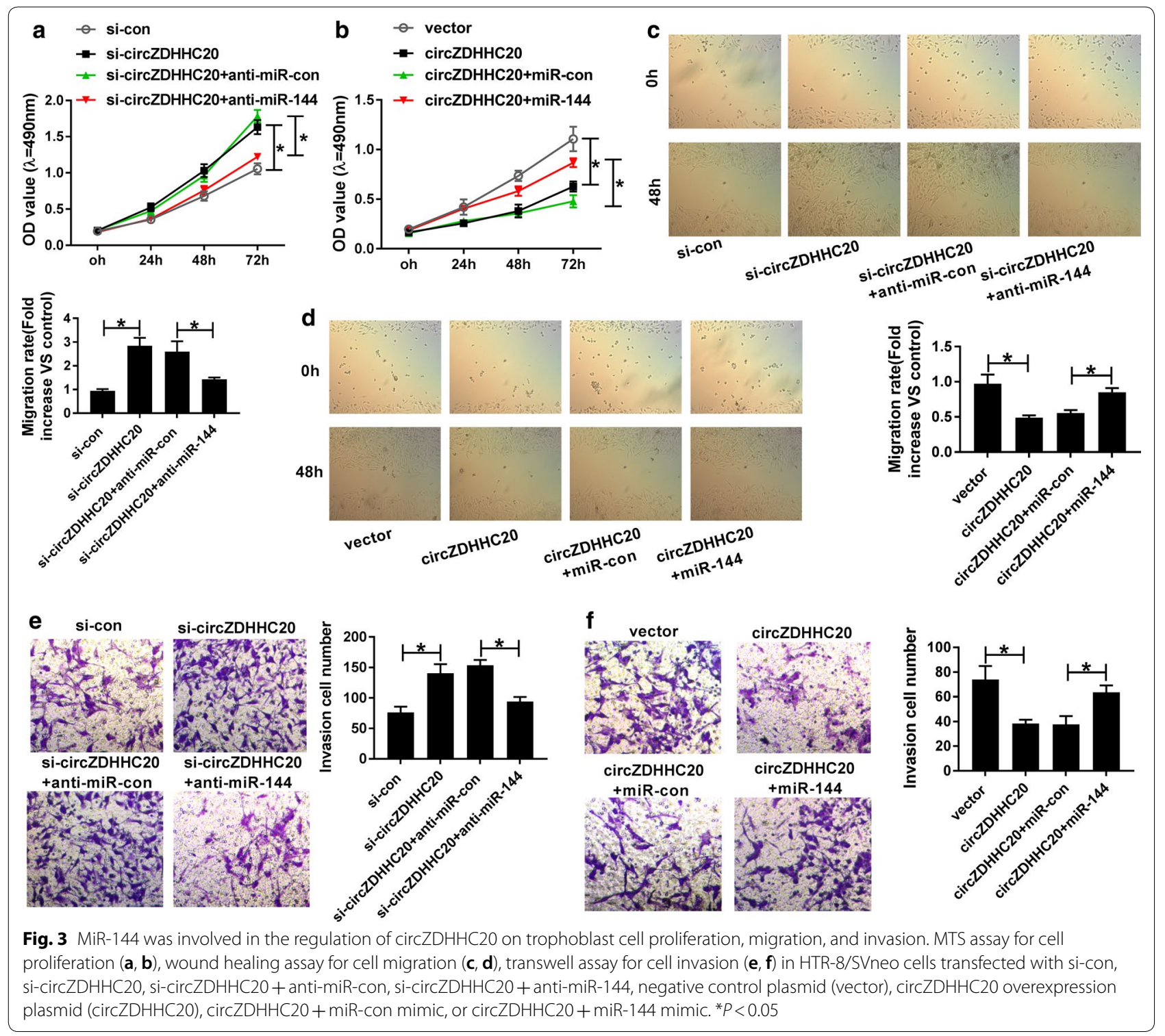

of PE [10, 20]. However, the potential function and underlying mechanisms of these circRNAs in PE development are not yet well understood. The database of online software algorithms predicted a possible ceRNA network of the circZDHHC20/miR-144/GRHL2 axis in PE. The present work had led to the identification of circZDHHC20 that suppressed the proliferation, migration, and invasion in trophoblast cells through sponging miR-144 and up-regulating GRHL2.

In the current project, our data firstly validated a significant up-regulation of circZDHHC20, consistent with previous work [10]. To verify that circZDHHC20 was indeed circular transcript, we carried out RNase $\mathrm{R}$ assay and reverse transcription experiments with
Oligo $(\mathrm{dT})_{18}$ primers on account of the inherent stability by their exonuclease resistance and the depletion of the $3^{\prime}$ pA tail, respectively. As expected, our data indicated that circZDHHC20 was resistant to RNase $\mathrm{R}$ digestion, and its level was lower when Oligo $(\mathrm{dT})_{18}$ primers were used. Since circRNAs prominently located in the cytoplasm could interact with available miRNAs [16, 18], we then validated the subcellular localization of circZDHHC20 in HTR-8/SVneo cells, and our data demonstrated that circZDHHC20 was predominantly present in the cytoplasm. To investigate the role of circZD$\mathrm{HHC} 20$ in PE pathogenesis, loss-of-function and gainof-function experiments were performed. We were first to uncover that circZDHHC20 overexpression 


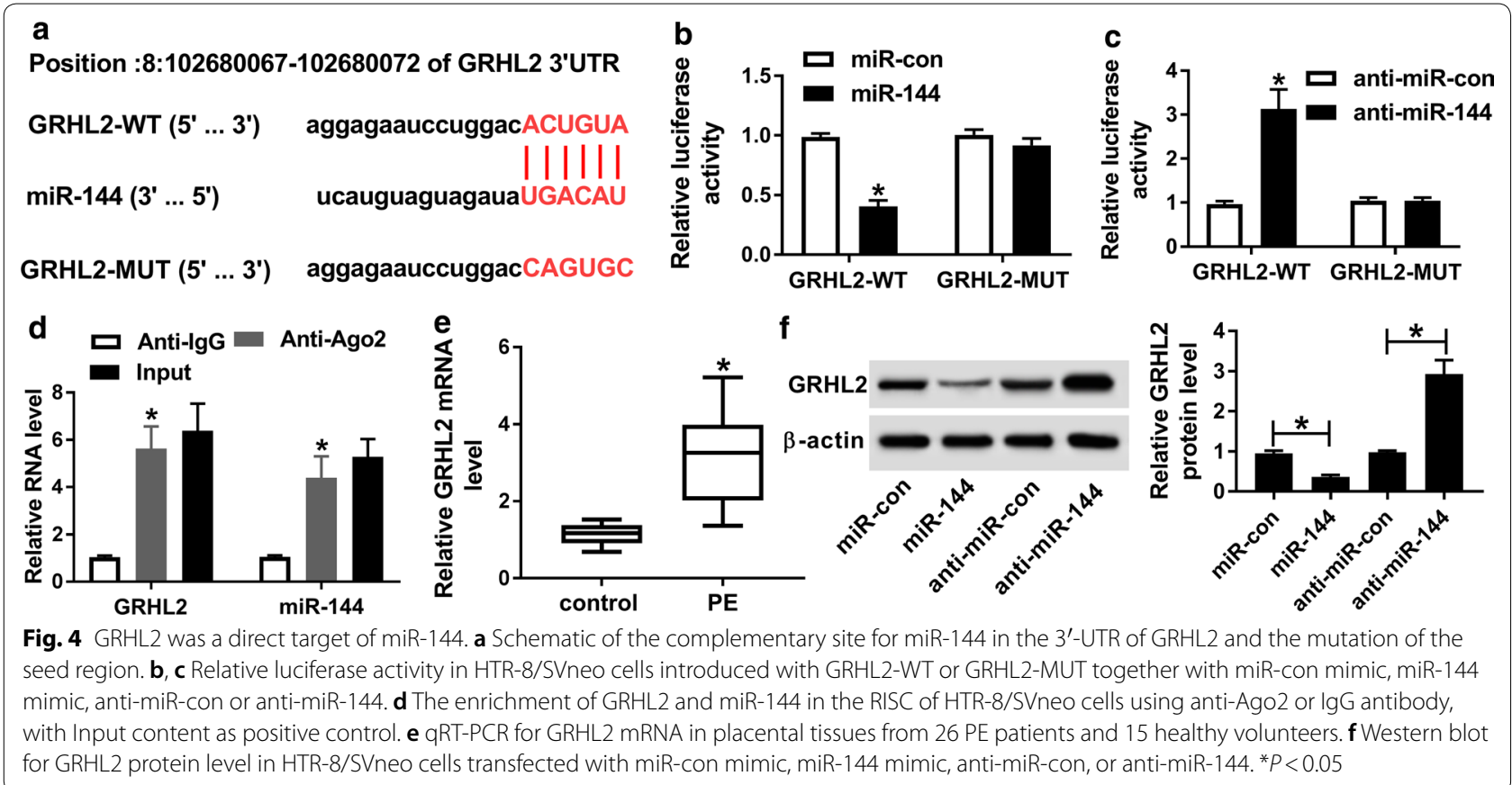

$\mathbf{a}$

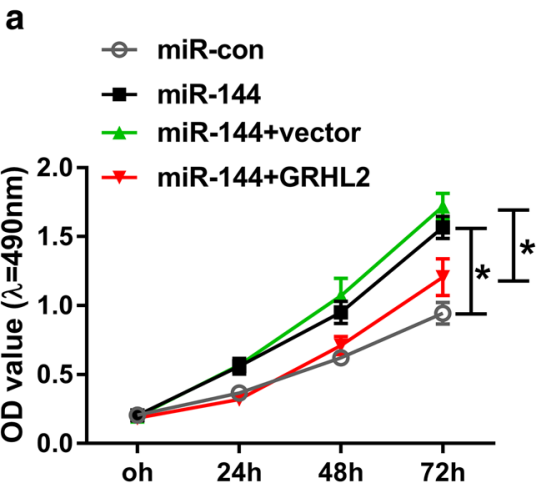

b $\quad \theta$ anti-miR-con

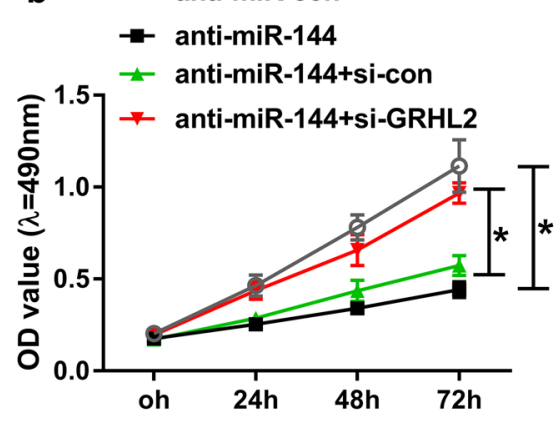

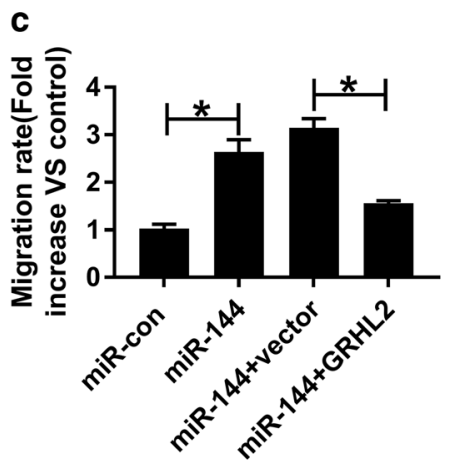

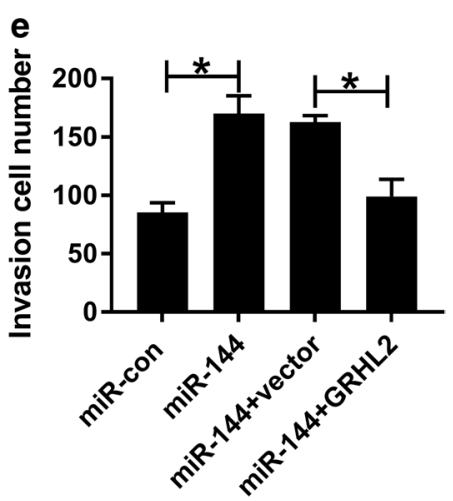

d

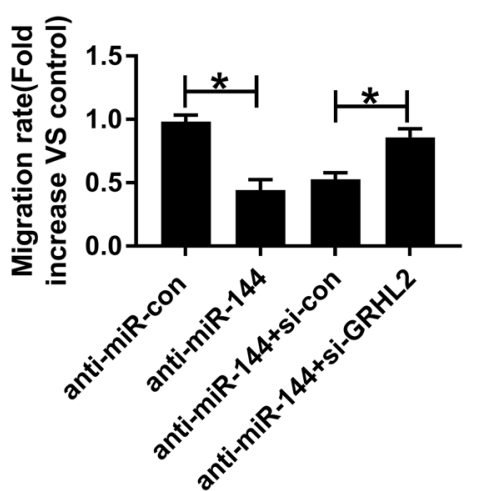

f

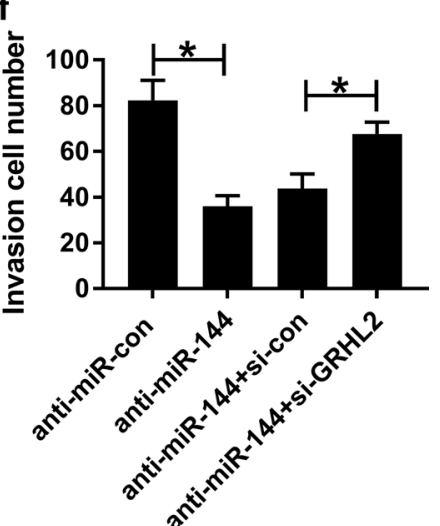

Fig. 5 GRHL2 mediated the regulatory effects of miR-144 on trophoblast cell proliferation, migration and invasion. Cell proliferation by MTS assay $(\mathbf{a}, \mathbf{b})$, cell migration by wound healing assay $(\mathbf{c}, \mathbf{d})$, cell invasion by transwell assay $(\mathbf{e}, \mathbf{f})$ in HTR-8/SVneo cells transfected with miR-con mimic, miR-144 mimic, miR-144 mimic + negative control plasmid (vector), miR-144 mimic + GRHL2 overexpression plasmid, anti-miR-con, anti-miR-144, anti-miR-144 + si-con, or anti-miR-144 + si-GRHL2. ${ }^{*} P<0.05$ 


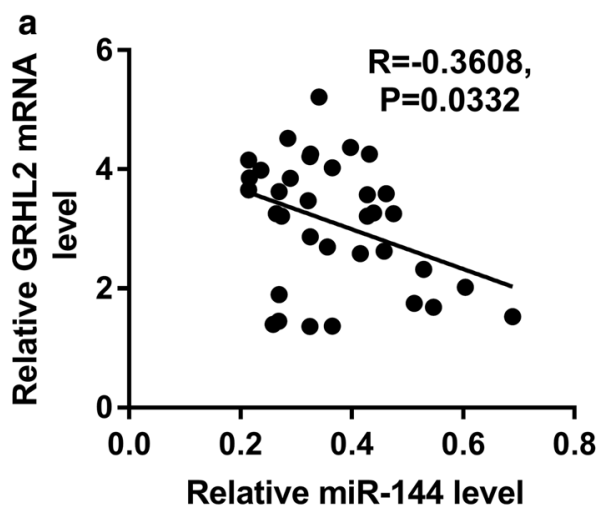

C

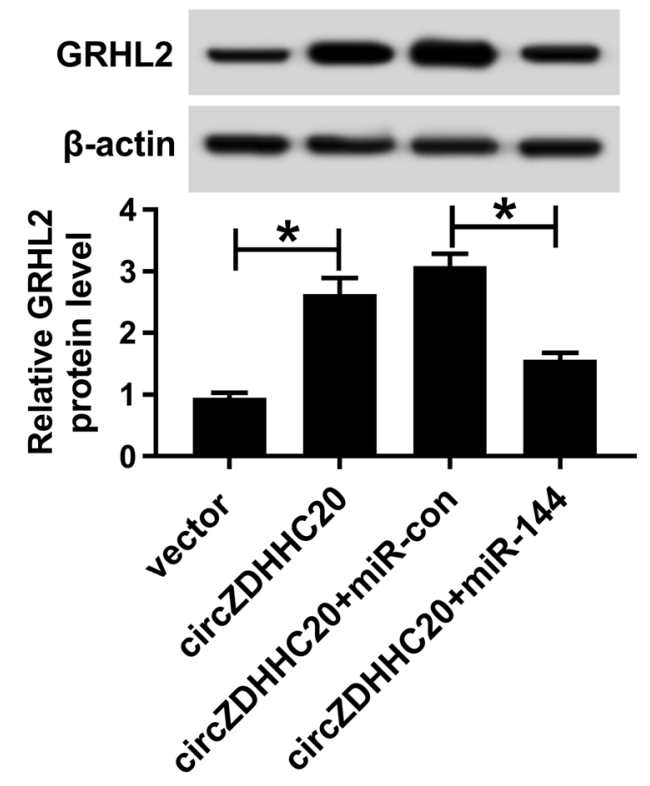

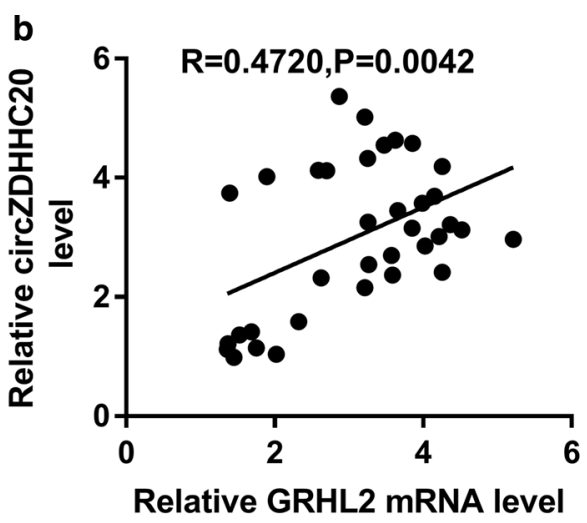

d

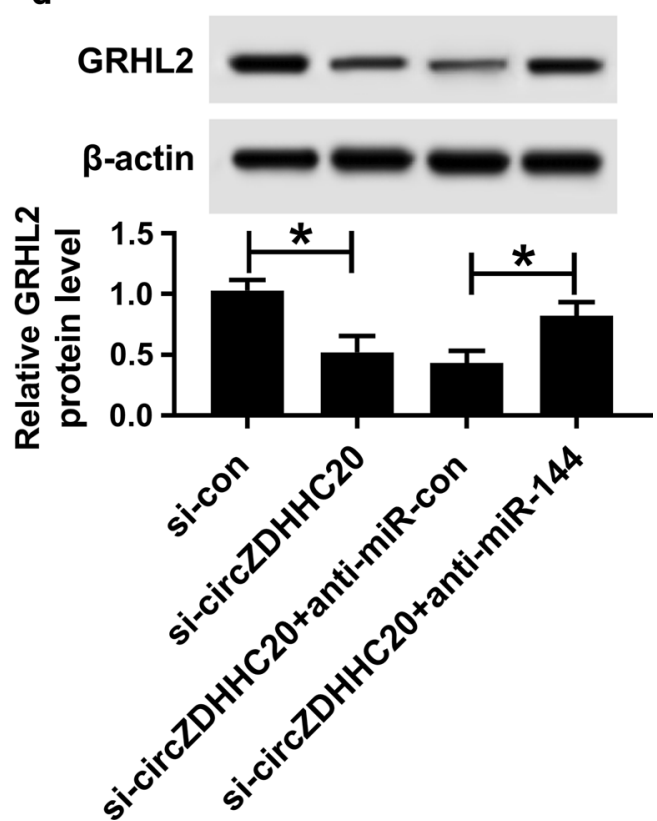

Fig. 6 CircZDHHC20 regulated GRHL2 expression through sponging miR-144. Correlations between GRHL2 mRNA expression and miR-144 level (a) or circZDHHC20 level (b) in 26 placental tissues from PE patients using the Spearman test. $\mathbf{c}$, $\mathbf{d}$ GRHL2 protein level by western blot in HTR-8/SVneo cells transfected with negative control plasmid (vector), circZDHHC20 overexpression plasmid (circZDHHC20), circZDHHC20 + miR-con mimic, circZDHHC20 + miR-144 mimic, si-con, si-circZDHHC20, si-circZDHHC20 + anti-miR-con or si-circZDHHC20 + anti-miR-144. ${ }^{*} P<0.05$

significantly retarded trophoblast cell proliferation, migration, and invasion, while its deficiency exhibited opposite effects. Increasing evidence has demonstrated that an increase in trophoblast migration/invasion restraining factors contributes to $\mathrm{PE}$ aggressiveness [21]. Based on the above, highly expressed circZDHHC20 was associated with PE pathogenesis.

Of particular interest, circRNAs regulate the abundance of specific miRNAs through mechanisms including sequestration. To confirm whether circZDHHC20 could act as miRNAs sponges, the CircInteractome computational method was used. Among these candidates, miR-144 was selected for further exploration owing to its significant down-regulation in PE placenta and maternal plasma [13-15]. As for miR-144, it was reported to regulate tumor cell proliferation, migration and invasion in a series of human cancers, such as breast cancer, pancreatic cancer, and nasopharyngeal carcinoma [22-24]. In the present work, we firstly validated that circZDHHC20 sequestered miR-144 through acting as a miR-144 sponge. Our data also indicated that miR-144 level was reduced and inversely correlated with circZDHHC20 expression in PE placental tissues. Moreover, miR-144 overexpression enhanced trophoblast cell proliferation, migration, and invasion, and its silencing showed opposite effects, in line with an earlier study [15]. 
More importantly, our results substantiated that miR-144 mediated the regulation of circZDHHC20 on proliferation, migration, and invasion in trophoblast cells.

Then, we used online software microT-CDS to help identify the molecular targets of miR-144. Interestingly, GRHL2 harbored a putative complementary sequence for miR-144. GRHL2 has been established as a tumor server through the regulation of cell proliferation, migration, and invasion in multiple cancers, including ovarian cancer and gastric cancer $[25,26]$. Moreover, GRHL2 hampered the differentiation of keratinocyte through reducing related protein expression via epigenetic mechanism [27]. In the present research, we firstly uncovered that GRHL2 was a direct target of miR-144 in trophoblast cells. Our data by qRT-PCR also demonstrated a significant up-regulation of GRHL2 level in PE placenta. Moreover, we validated that miR-144 regulated trophoblast cell proliferation, migration, and invasion by targeting GRHL2. Additionally, our data underscored that circZDHHC20 regulated GRHL2 repression through acting as a sponge of miR-144. Similar to our findings, Shen et al. reported that circTRNC18 accelerated PE progression via sponging miR-762 and modulating GRHL2 expression [9].

\section{Conclusion}

In conclusion, our study suggested that circZDHHC20 inhibited the proliferation, migration, and invasion in trophoblast cells partially through targeting miR-144/ GRHL2 axis. Our present work provided a novel mechanism involved in PE pathogenesis.

\section{Acknowledgements \\ None.}

\section{Author's contributions}

$B Z, X Z$ and $C Y$ conceived and designed the study; $X Z$, TL collected and analyzed the data; RX and JZ contributed reagents/materials/analysis tools; and $Y L$ wrote the paper. All authors read and approved the final manuscript.

\section{Funding}

None.

\section{Availability of data and materials}

All data generated or analyzed during this study are included in this published article.

\section{Ethics approval and consent to participate}

Specimen collection and analysis processes were approved by the Ethics Committee of The Second Affiliated Hospital, University of South China.

\section{Consent for publication}

Not applicable.

\section{Competing interests}

The authors declare that they have no competing interests.

\section{Author details}

${ }^{1}$ Department of Obstetrics and Gynecology, The Second Affiliated Hospital, University of South China, Hengyang, Hunan, China. ${ }^{2}$ Department of Ultrasonography, The Second Affiliated Hospital, University of South China, Hengyang, Hunan, China. ${ }^{3}$ Department of Obstetrics and Gynecology, Guangdong Maternal and Child Health Hospital, Guangzhou, Guangdong, China. ${ }^{4}$ Department of Obstetrics and Gynecology, The First Affiliated Hospital, University of South China, No. 69 Chuanshan Road, Hengyang 421001, Hunan, China.

Received: 13 November 2019 Accepted: 4 January 2020

Published online: 13 January 2020

\section{References}

1. Phipps E, Prasanna D, Brima W, Jim B. Preeclampsia: updates in pathogenesis, definitions, and guidelines. Clin J Am Soc Nephrol. 2016;11(6):1102-13.

2. Rana S, Lemoine E, Granger J, Karumanchi SA. Preeclampsia: pathophysiology, challenges, and perspectives. Circ Res. 2019;124(7):1094-112.

3. Lee CQ, Gardner L, Turco M, Zhao N, Murray MJ, Coleman N, Rossant J, Hemberger M, Moffett A. What is trophoblast? a combination of criteria define human first-trimester trophoblast. Stem Cell Rep. 2016;6(2):257-72

4. Lala PK, Nandi P. Mechanisms of trophoblast migration, endometrial angiogenesis in preeclampsia: the role of decorin. Cell Adhes Migr. 2016;10(1-2):111-25.

5. Qu S, Yang X, Li X, Wang J, Gao Y, Shang R, Sun W, Dou K, Li H. Circular RNA: a new star of noncoding RNAs. Cancer Lett. 2015;365(2):141-8.

6. Haque S, Harries LW. Circular RNAs (circRNAs) in health and disease. Genes. 2017;8(12):353.

7. Deng N, Lei D, Huang J, Yang Z, Fan C, Wang S. Circular RNA expression profiling identifies hsa_circ_0011460 as a novel molecule in severe preeclampsia. Pregnancy Hypertens. 2019;17:216-25.

8. Zhou W, Wang H, Yang J, Long W, Zhang B, Liu J, Yu B. Down-regulated circPAPPA suppresses the proliferation and invasion of trophoblast cells via the miR-384/STAT3 pathway. Biosci Rep. 2019. https://doi.org/10.1042/ BSR20191965.

9. Shen XY, Zheng LL, Huang J, Kong HF, Chang YJ, Wang F, Xin H. CircTRNC18 inhibits trophoblast cell migration and epithelial-mesenchymal transition by regulating miR-762/Grhl2 pathway in pre-eclampsia. RNA Biol. 2019;16(11):1565-73.

10. Jiang M, Lash GE, Zhao X, Long Y, Guo C, Yang H. CircRNA-0004904, CirCRNA-0001855, and PAPP-A: potential novel biomarkers for the prediction of preeclampsia. Cell Physiol Biochem. 2018;46(6):2576-86.

11. Enquobahrie DA, Abetew DF, Sorensen TK, Willoughby D, Chidambaram $\mathrm{K}$, Williams MA. Placental microRNA expression in pregnancies complicated by preeclampsia. Am J Obstet Gynecol. 2011;204(2):178.e12-21.

12. Lagana AS, Vitale SG, Sapia F, Valenti G, Corrado F, Padula F, Rapisarda AMC, D'Anna R. miRNA expression for early diagnosis of preeclampsia onset: hope or hype? J Matern Fetal Neonatal Med. 2018;31(6):817-21.

13. Jairajpuri DS, Malalla ZH, Mahmood N, Almawi WY. Circulating microRNA expression as predictor of preeclampsia and its severity. Gene. 2017:627:543-8.

14. Li H, Ge Q, Guo L, Lu Z. Maternal plasma miRNAs expression in preeclamptic pregnancies. BioMed Res Int. 2013. https://doi. org/10.1155/2013/970265.

15. Xiao J, Tao T, Yin Y, Zhao L, Yang L, Hu L. miR-144 may regulate the proliferation, migration and invasion of trophoblastic cells through targeting PTEN in preeclampsia. Biomed Pharmacother. 2017;94:341-53.

16. Hansen TB, Jensen TI, Clausen BH, Bramsen JB, Finsen B, Damgaard CK, Kjems J. Natural RNA circles function as efficient microRNA sponges. Nature. 2013;495(7441):384-8.

17. Hu X, Ao J, Li X, Zhang H, Wu J, Cheng W. Competing endogenous RNA expression profiling in pre-eclampsia identifies blood biomarker for early pre-eclampsia. Clin Epigenet. 2018;10:48.

18. Chen LL. The biogenesis and emerging roles of circular RNAs. Nat Rev Mol Cell Biol. 2016;17(4):205-11.

19. Iwakawa HO, Tomari Y. The functions of microRNAs: mRNA decay and translational repression. Trends Cell Biol. 2015;25(11):651-65. 
20. Qian Y, Lu Y, Rui C, Qian Y, Cai M, Jia R. Potential significance of circular RNA in human placental tissue for PATIENTS with preeclampsia. Cell. Physiol Biochem. 2016;39(4):1380-90.

21. Lala PK, Chakraborty C. Factors regulating trophoblast migration and invasiveness: possible derangements contributing to pre-eclampsia and fetal injury. Placenta. 2003;24(6):575-87.

22. Yin Y, Cai J, Meng F, Sui C, Jiang Y. MiR-144 suppresses proliferation, invasion, and migration of breast cancer cells through inhibiting CEP55. Cancer Biol Ther. 2018;19(4):306-15.

23. Liu S, Luan J, Ding Y. miR-144-3p targets FosB proto-oncogene, AP-1 transcription factor subunit (FOSB) to suppress proliferation, migration, and invasion of PANC-1 pancreatic cancer cells. Oncol Res. 2018;26(5):683-90.

24. Zhang LY, Ho-Fun Lee V, Wong AM, Kwong DL, Zhu YH, Dong SS, Kong KL, Chen J, Tsao SW, Guan XY, et al. MicroRNA-144 promotes cell proliferation, migration and invasion in nasopharyngeal carcinoma through repression of PTEN. Carcinogenesis. 2013;34(2):454-63.
25. Faddaoui A, Sheta R, Bachvarova M, Plante M, Gregoire J, Renaud MC, Sebastianelli A, Gobeil S, Morin C, Ghani K, et al. Suppression of the grainyhead transcription factor 2 gene (GRHL2) inhibits the proliferation, migration, invasion and mediates cell cycle arrest of ovarian cancer cells. Cell Cycle. 2017;16(7):693-706.

26. Xiang J, Fu X, Ran W, Wang Z. Grhl2 reduces invasion and migration through inhibition of TGFbeta-induced EMT in gastric cancer. Oncogenesis. 2017;6(1):e284.

27. Chen W, Xiao Liu Z, Oh JE, Shin KH, Kim RH, Jiang M, Park NH, Kang MK. Grainyhead-like 2 (GRHL2) inhibits keratinocyte differentiation through epigenetic mechanism. Cell Death Dis. 2012;3:e450.

\section{Publisher's Note}

Springer Nature remains neutral with regard to jurisdictional claims in published maps and institutional affiliations.
Ready to submit your research? Choose BMC and benefit from:

- fast, convenient online submission

- thorough peer review by experienced researchers in your field

- rapid publication on acceptance

- support for research data, including large and complex data types

- gold Open Access which fosters wider collaboration and increased citations

- maximum visibility for your research: over $100 \mathrm{M}$ website views per year

At BMC, research is always in progress.

Learn more biomedcentral.com/submissions 